\title{
Praziquantel, levamisol e diflubenzuron no controle de Dolops carvalboi (Crustacea: Branchiura) e Anacanthorus penilabiatus (Monogenea: Dactylogyridae) em Piaractus mesopotamicus Holmberg, 1887 (Osteichthyes: Characidae)
}

Praziquantel, levamisole and diflubenzuron in the control of Dolops carvalhoi (Crustacea: Branchiura) and Anacanthorus penilabiatus (Monogenea: Dactylogyridae) in Piaractus mesopotamicus

Holmberg, 1887 (Osteichthyes: Characidae)

Sergio Henrique Canello Schalch ${ }^{1 *}$; Flávio Ruas de Moraes ${ }^{2,3}$; Vando Edésio Soares ${ }^{3}$

${ }^{1}$ Agência Paulista de Tecnologia e dos Agronegócios, Pesca e Aquicultura, Votuporanga - SP, Brasil

${ }^{2}$ Centro de Aquicultura da UNESP - CAUNESP, Universidade Estadual Paulista - UNESP

${ }^{3}$ Centro de Pesquisas em Sanidade Animal, Universidade Estadual Paulista - UNESP

Recebido em 31 de Maio de 2008

Aceito em 27 de Fevereiro de 2009

\section{Resumo}

Neste trabalho, avaliou-se a eficácia antiparasitária do praziquantel, levamisol e diflubenzuron administrados via oral, adicionados à ração, para pacus (Piaractus mesopotamicus) infectados por Anacanthorus penilabiatus e Dolops carvalhoi. Foram utilizadas 19 caixas d'água de $300 \mathrm{~L}$ de capacidade, comportando 28 peixes cada. Os tratamentos foram feitos misturando os princípios ativos nas dietas. A intensidade parasitária e eficácia foram avaliadas 1 dia antes e 3, 7 e 15 dias após o início da alimentação com ração contendo diflubenzuron, levamisol e praziquantel isolados ou associados em diferentes concentraçóes por 7 dias. Os resultados da eficácia terapêutica sugerem que, isoladamente ou associado com levamisol e praziquantel, o diflubenzuron é eficiente contra o crustáceo $D$. carvalhoi, demonstrando que a eficácia dos tratamentos nos dias 3,7 e 15 variou de 96,2 a 100\%. Contra os monogenóides, as drogas não apresentaram eficácia satisfatória. Os resultados sugerem o uso do diflubenzuron para o controle de $D$. cavalhoi em peixes de cativeiro e em condiçóes de quarentenário.

Palavras-chave: controle, parasitos, dieta, peixes.

\begin{abstract}
This assay evaluated the control efficacy of diflubenzuron, praziquantel and levamisole added to the diet of pacu (Piaractus mesoptamicus) infected with Anacanthorus penilabiatus and Dolops carvalhoi. 19 water tanks of $300 \mathrm{~L}$ capacity were utilized with 28 fish in each one. The treatments were made by mixing the active principles in the diet. The experiment was evaluated in four harvests done 1 day before and 3, 7 and 15 days after the treatment. The medicated feeding was applied for 7 days. The results of efficacy suggest that the diflubenzuron alone or associated with levamisole and praziquantel was efficient against the crustacean $D$. carvalho $i$ and the efficacy in the 3,7 and 15 days evaluations ranged from 96,2 to $100 \%$. Against the monogenean the drugs did not present efficacy. The results suggest the use of diflubenzuron for the control of $D$. carvalhoi in captive fishes in special conditions.
\end{abstract}

Keywords: control, parasites, diet, fishes.

\section{Introdução}

A criação intensiva torna os peixes mais susceptíveis às enfermidades em decorrência do estresse e da má qualidade da água, que favorece a proliferação de organismos com potencial patogênico, acarretando surtos de doenças infecciosas e parasitárias (MORAES; MARTINS, 2004).

\footnotetext{
*Autor para correspondência: Sérgio Henrique Canello Schalch Pólo Regional Noroeste Paulista, Agência Paulista de Tecnologia e dos Agronegócios, Pesca e Aquicultura, Rod. Péricles Belini, km 121, CP 61, CEP 15500-970 Votuporanga - SP, Brasil; e-mail: sschalch@apta.sp.gov.br Apoio: FAPESP, processo: 03/01881-9
}

Crustáceos da subfamília Branchiura, como Argulus sp. e Dolops sp., parasitam a superfície corporal, nadadeiras e brânquias de várias espécies de peixes silvestres e de cativeiro (EIRAS, 1994; PAVANELLI et al., 2002). Como são histófagos, a penetraçấo do seu aparelho bucal causa dano mecânico ao hospedeiro e a injeção de enzimas tóxicas determina inflamação local (SHIMURA, 1983). A agressão do Dolops sp. é severa, visto que seu aparelho de fixação é dotado de ganchos e, ao mudarem constantemente de lugar, ampliam os danos 
ao hospedeiro. Nos locais agredidos, observam-se hemorragias puntiformes, excesso de muco e hiperpigmentaçáo na pele. Nas brânquias há hipertrofia com hiperplasia do epitélio de revestimento e de células mucóides, além de focos necróticos, que prejudicam as trocas gasosas e iônicas. Os parasitos atuam como vetores de bacterioses e viroses de importância (SHIMURA, 1983). Os Dactylogyridae têm forma alongada, são monoxêmicos, com exceção do Diplozoon sp., favorecendo as grandes infestaçôes. Ao liberarem seus ovos dão origem aos oncomiracídeos infectantes, e os monogenóides adultos alimentam-se do epitélio cutâneo e branquial, causando processo irritativo e anorexia (RHODE, 1993).

No Brasil causam doença em pacus (Piaratus mesopotamicus), tambaquis (Colosssoma macropomum) e no híbrido tambacu. Nos filamentos branquiais, causam petéquias, hiperplasia epitelial, inflamação e edema subepitelial com ruptura de células pilares (MARTINS; ROMERO, 1996).

O diflubenzuron (1-(4-clorofenil)-3-(2-6-diflurobenzoil) uréia) (DFB) é potente regulador de crescimento de artrópodes, pois interfere na síntese de quitina durante a fase de muda de estágios imaturos (EISLER, 1992). Hosberg e Hoy (1991) relataram seu potencial contra infestaçôes causadas pelo "piolho de salmáo" (Caligus sp.), sendo aprovado pelo EPA (Environmental Protection Agency) (TANNER; MOFFETT, 1995). A imersão prolongada dos peixes em $0,03 \mathrm{mg}$ de diflubenzuron por litro de água é a mais indicada, segundo NOGA (1996).

O praziquantel é usado em medicina humana para controlar infecçōes por trematódeos e cestóides (REDMAN et al., 1996), e o levamisol para nematóides (ROBERTSON; MARTIN, 1993). Informaçóes sobre o uso desses princípios ativos no controle de monogenéticos são escassos. Schmahl e Mehlhorn (1985) demonstraram a efetividade de tratamento com praziquantel para Dactylogyrus vastator, D. extensus e Diplozoon paradoxum. Em carpas (Cyprinus carpio), nas doses de 0;1,0; 5,0;10,0 e 100,0 mg. $\mathrm{L}^{-1}$, a dose de $1,0 \mathrm{mg} . \mathrm{L}^{-1}$ causou danos irreversíveis no tegumento do monogenóide após 30 minutos de exposição. Os resultados sugerem que a utilizaçáo de $10 \mathrm{mg} \cdot \mathrm{L}^{-1}$, durante 3 horas, à temperatura de $22^{\circ} \mathrm{C}$, foi eficaz para controlar esses parasitos.

Hirazawa et al. (2000) verificaram eficácia do praziquantel (2.000 e $4.000 \mathrm{mg} \cdot \mathrm{kg}^{-1}$ de raçáo) para peixes parasitados por larvas de monogenóides (Heterobothrium okamotoi).

O levamisol, forma levógira do tetramisol, é um anti-helmíntico do grupo dos imidotiazóis, e seu provável efeito no parasito concentra-se na estimulação dos gânglios parassimpáticos e simpáticos (GUSTAFSSON et al., 1987). Sua ação em peixes não foi encontrada na literatura que foi possível compulsar.

Diante do exposto, este ensaio teve como objetivo avaliar a eficácia do praziquantel, levamisol e diflubenzuron adicionados à ração peletizada para $P$. mesopotamicus, no controle de Dolops carvalhoi e A. penilabiatus.

\section{Material e Métodos}

Os ensaios foram conduzidos na Universidade Estadual Paulista Júlio de Mesquita Filho, campus de Jaboticabal $\left(21^{\circ} 15^{\prime} 22^{\prime \prime} \mathrm{S}\right.$ e $48^{\circ} 18^{\prime} 58^{\prime \prime} \mathrm{W}$, altitude de $\left.595 \mathrm{~m}\right)$. Para infestaçôes de monogenóides foram utilizados exemplares adultos de P. mesopotamicus, oriundos de pesque-pague e/ou pisciculturas comerciais, após prévio exame parasitológico. Esses animais foram acondicionados junto com os mais jovens em tanques de alvenaria de $16 \mathrm{~m}^{3}$, por duas semanas, tempo necessário para o monogenóide completar seu ciclo de vida e infectar os peixes jovens. (PELLITERO, 1988; THATCHER, 1991).

Desse lote inicial de 1.400 exemplares de pacus (P. mesopotamicus Holmberg, 1887) jovens, provenientes do Centro de Aquicultura da UNESP - CAUNESP e oriundos da mesma desova, foram selecionados 532 peixes pesando entre 40 e $100 \mathrm{~g}$ após biometria. Esses peixes selecionados foram transferidos para 19 caixas d'água de plástico com $300 \mathrm{~L}$ de capacidade. Em cada caixa foram acondicionados 28 peixes que foram infectados por $A$. penilabiatus.

Para infestação com $D$. carvalhoi, foram utilizadas mais 6 caixas d'água de $300 \mathrm{~L}$. Para isto foram transportados de um pesque-pague da regiáo exemplares de $P$. mesopotamicus parasitados por adultos até o CAUNESP infestados por D. carvalhoi adultos. Após reprodução, incubação e crescimento (10 a 50 dias), os parasitos permaneceram nas 6 caixas até o estágio pré-adultos quando foram utilizados para infestação. $\mathrm{O}$ número de parasitos colocados nas 19 caixas para a obtenção de infestação não muito severa para o ensaio foi de 400 espécimes. No entanto, foi observado que os peixes jovens se alimentaram de alguns exemplares antes dos parasitos se fixarem ao hospedeiro.

Os tratamentos foram ministrados adicionando-se à ração basal (Tabela 1) os princípios ativos praziquantel - PZQ, levamisol - LVM e diflubenzuron - DFB. Os peixes foram distribuídos em grupos ao acaso e alimentados com a ração contendo esses fármacos.

À ração contendo $26 \%$ de proteína bruta e $4.150 \mathrm{kcal}$ de energia bruta, foi adicionado o praziquantel, levamisol e diflubenzuron de acordo com o delineamento proposto e, a seguir, peletizada. O diflubenzuron (25\%) e o praziquantel (98\%) foram utilizados na forma de pó, e o levamisol na forma líquida (23\%). A presença dos princípios ativos na ração foi avaliada por análise de cromatografia líquida (HPLC) (LABTEC - Guabi).

Os peixes foram alimentados duas vezes ao dia, $3 \%$ da biomassa, por 7 dias. A avaliação da intensidade parasitária foi realizada antes e 3, 7 e 15 dias após por exame parasitológico (THATCHER, 1991), determinando-se a eficácia dos tratamentos. Em cada tempo foram colhidos 7 peixes de cada tratamento, diminuindo-se proporcionalmente o volume de água. Diariamente foi determinada a temperatura, $\mathrm{pH}$ e oxigênio dissolvido e, a cada 3 dias, a concentração de amônia. Todas as características hídricas permaneceram dentro de parâmetros adequados para os peixes (SIPAÚBA-TAVARES, 1995). Em cada colheita, os peixes foram anestesiados em soluçáo aquosa de benzocaína 1:10.000 (NOGA, 1996), medidos e pesados. 
O percentual de eficácia dos tratamentos foi calculado obedecendo à Equação 1:

Eficácia $(\%)=\frac{x-y}{x} \times 100$

onde: $\mathrm{x}=$ média do número de parasitos do grupo controle; $\mathrm{e}$ $\mathrm{y}=$ média do número de parasitos do grupo tratado.

Os resultados das contagens de monogenóides foram transformados em $\log (x+1)$ segundo Little e Hills (1978) e analisados em delineamento inteiramente casualizado. Das contagens transformadas em $\log (\mathrm{x}+1)$ foram obtidas as médias aritméticas $\left(=10^{\left[\sum \log (x+1) / n\right]}-1\right)$ dos tratamentos, que foram confrontadas pelo teste de Scheffé, ao nível de 95\% de confiança. Tais análises foram efetuadas utilizando-se o Software SAS versão 8.2 (SAS, 1999-2001).

\section{Resultados e Discussáo}

O resultado da dosagem de medicamentos na ração está expresso na Tabela 1. Da sua análise, verifica-se que o praziquantel apresentou níveis próximos ao adicionado à ração, assim como o diflubenzuron. No caso do levamisol, em quase todas as concentraçôes, ocorreram perdas. Com a alteração na dosagem esperada de levamisol, não foi possível observar o efeito desejado, como proposto inicialmente. Para o diflubenzuron, a perda da substância só foi verificada nos tratamentos em que se utilizou a dose mais alta, mas sem interferência no resultado final.

Os resultados apresentados na Figura 1 e Tabela 2 permitem verificar que os tratamentos de 1 a 4 (praziquantel associado com diflubenzuron) e de 5 a 7 (levamisol combinado com diflubenzuron) tiveram relativa eficácia contra $A$. penilabitus nos
3 tempos de observação. As menores eficácias foram observadas nos grupos tratados com praziquantel e levamisol, isoladamente ou em associação.

Há dificuldades na terapêutica contra monogenóides no ambiente de criação em cativeiro, em consequência da baixa eficácia das drogas e alto nível de estresse dos peixes (PIRONET; JONES, 2000). Todavia, há boa eficiência no uso de $0,5 \mathrm{mg} . \mathrm{L}^{-1}$ de água de praziquantel em banhos de 30 minutos, sendo interessante a inclusão deste produto em dietas para peixes infectados por Haliotrema abaddon, pois o fármaco age especificamente contra plathelmintos e é insolúvel em água (PIRONET; JONES, 2000). Utilizando-se $2 \mathrm{mg}$. $\mathrm{L}^{-1}$ de água de praziquantel por trinta horas, na forma de banhos, Stephens et al. (2003) observaram efetividade $(\mathrm{p}<0,05) \mathrm{em}$ Glaucosoma hebraicum infectado contra $H$. abaddon. Esses resultados, no entanto, representam parasitos e peixes criados em ambiente salino, fatores que não recomendam comparação com os achados deste trabalho realizado em água doce e clima tropical.

Neste ensaio, a associação destes produtos, praziquantel e levamisole, adicionados à ração, apresentou baixa eficiência contra monogenóides (Tabela 2). O levamisol é anti-helmíntico de amplo espectro, com atividade contra diversos tricostrongilídeos, estrongilídeos e helmintos pulmonares e vasculares, nas formas adultas e jovens. O praziquantel, em doses relativamente baixas exerce elevada atividade contra cestóides e todas as tênias (LAMMLER; GIESSEN, 1977). Neste ensaio, investigou-se os benefícios da associação de levamisol e praziquantel, na busca de melhor eficácia contra os helmintos monogenóides, mas os resultados não permitem a indicaçáo das referidas drogas para o controle dessa parasitose. Entretanto, quando o praziquantel foi associado ao diflubenzuron (952 e $1.243 \mathrm{mg} \cdot \mathrm{kg}^{-1}$ de raçáo), observou-se eficácia próxima a 90\%, que pode ser atribuída ao efeito do diflubenzuron (Figura 1).

Tabela 1. Resultados das concentraçóes de diflubenzuron - DFB, praziquantel - PZQ e levamisol - LVM na ração determinadas por cromatografia líquida (HPLC).

\begin{tabular}{|c|c|}
\hline Níveis reais de princípios ativos adicionados na raçáo & Níveis de princípios ativos detectados na raçáo (HPLC) \\
\hline T-1 - PZQ 1000 mg + 1000 mg DFB & $\mathrm{T}-1-\mathrm{PZQ} 950 \mathrm{mg}+941 \mathrm{mg} \mathrm{DFB}$ \\
\hline T-2 - PZQ $1000 \mathrm{mg}+2000 \mathrm{mg}$ DFB & T-2 - PZQ $952 \mathrm{mg}+1243 \mathrm{mg}$ DFB \\
\hline T-4 - PZQ $2000 \mathrm{mg}+2000 \mathrm{mg}$ DFB & T-4 - PZQ $2010 \mathrm{mg}+1273 \mathrm{mg}$ DFB \\
\hline T-5 - LVM $1000 \mathrm{mg}+1000 \mathrm{mg}$ DFB & T-5 - LVM $380 \mathrm{mg}+949 \mathrm{mg}$ DFB \\
\hline T-8 - LVM $2000 \mathrm{mg}+2000 \mathrm{mg}$ DFB & T-8 - LVM $436 \mathrm{mg}+1291 \mathrm{mg}$ DFB \\
\hline T-9 - DFB $1000 \mathrm{mg}$ & T-9 - DFB $935 \mathrm{mg}$ \\
\hline T-10 - DFB $2000 \mathrm{mg}$ & $\mathrm{T}-10-\mathrm{DFB} 1254 \mathrm{mg}$ \\
\hline T-11 - LVM $1000 \mathrm{mg}+1000 \mathrm{mg}$ PZQ & T-11 - LVM $350 \mathrm{mg}+958 \mathrm{mg}$ PZQ \\
\hline T-14 - LVM $2000 \mathrm{mg}+2000 \mathrm{mg}$ PZQ & T-14 - LVM $438 \mathrm{mg}+1152 \mathrm{mg}$ PZQ \\
\hline T-15 - PZQ $1000 \mathrm{mg}$ & T-15 - PZQ 977 mg \\
\hline T-16 - PZQ $2000 \mathrm{mg}$ & T-16 - PZQ 1964 mg \\
\hline T-17 - LVM $1000 \mathrm{mg}$ & T-17 - LVM $362 \mathrm{mg}$ \\
\hline T-18 - LVM $2000 \mathrm{mg}$ & T-18 - LVM $640 \mathrm{mg}$ \\
\hline
\end{tabular}




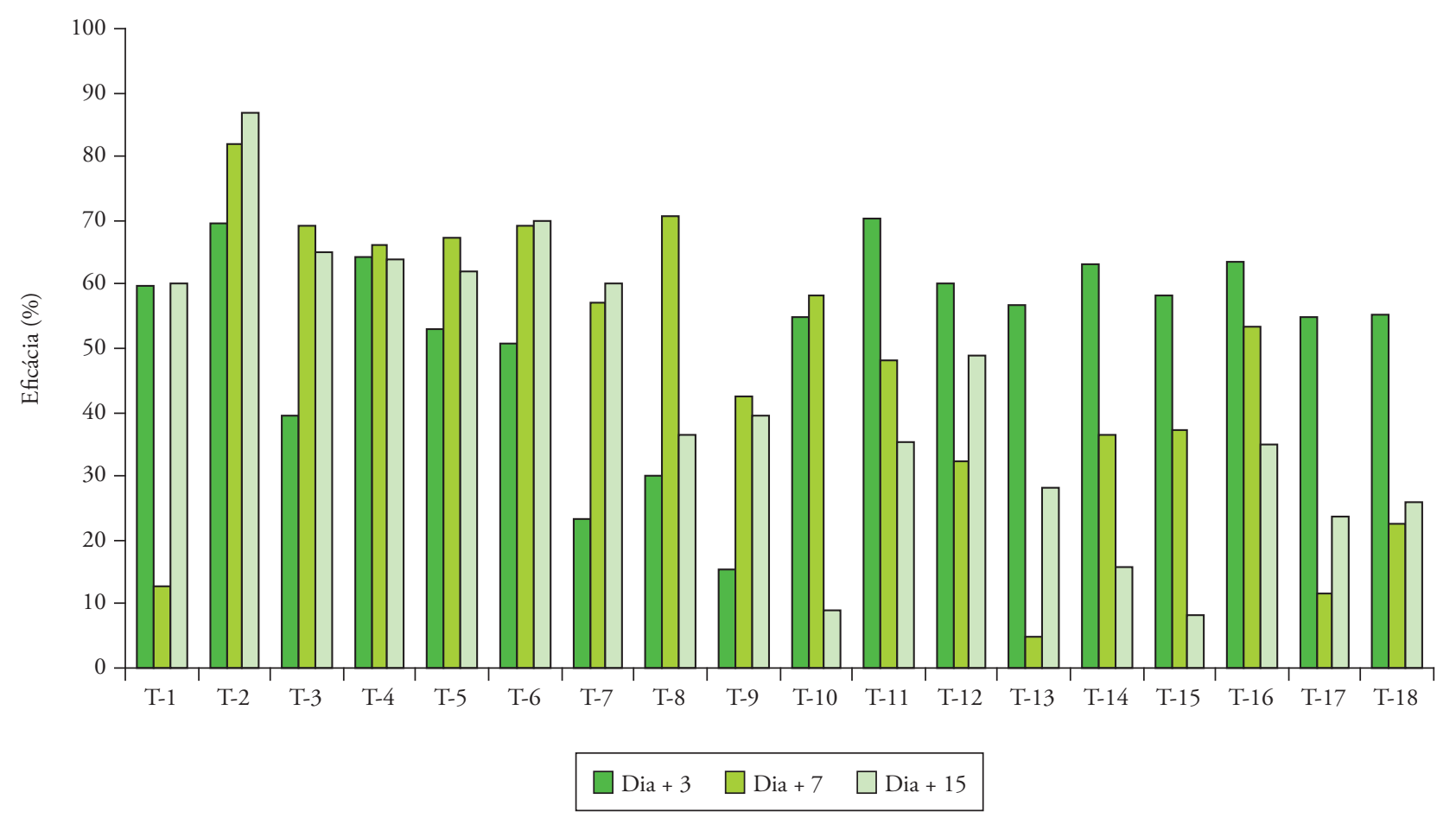

Figura 1. Percentual de eficácia dos antiparasitários contra o helminto Anacanthorus penilabitus após tratamento com praziquantel e diflubenzuron (T-1 a T-4); levamisol e diflubenzuron (T-5 a T-8); diflubenzuron (T-9 e T-10); levamisol e praziquantel (T-11 a T-14); praziquantel (T-15 e T-16) ou levamisol pela ração (T-17 e T-18) em avaliaçóes realizadas após 3, 7 e 15 dias, em Piaractus mesopotamicus.

Pela Tabela 3 verifica-se que o número médio de $D$. carvalhoi apresentou redução parcial, na segunda avaliação, e total na terceira. Não foram observados parasitos na superfície corporal dos peixes e nem nas caixas de água ao término do ensaio. Os peixes apresentavam-se sadios e com coloração brilhante durante e após os tratamentos. Verificou-se que, após 3, 7 e 15 dias, o diflubenzuron apresentou alta eficácia contra a forma pré-adulta deste crustáceo. Quando associado ao praziquantel e ao levamisol, a eficácia também foi elevada (Tabela 4). Eficácia de 97,2\% foi obtida por Schalch et al. (2005), que utilizaram banhos terapêuticos de trinta minutos com diflubenzuron (2 mg. $\mathrm{L}^{-1}$ de água) em pacus naturalmente infestados pelo mesmo parasito. $\mathrm{O}$ diflubenzuron adicionado à ração para controle de "piolho de salmão" (Caligus elongatus, Lepotheirus salmonis) sugere o seu uso em águas abertas, pois o produto não se dispersa na água, sendo seus metabólitos predominantemente excretados nas fezes e depositados para sedimentação e degradação (ERDAL, 1997).

Os resultados deste trabalho mostram que os peixes mantiveram-se em bom crescimento, e o consumo foi suficiente para eliminar o crustáceo com o uso de diflubenzuron na ração na concentração de 0,935 a 0,968 e 1.237 a $1.291 \mathrm{mg} \cdot \mathrm{kg}^{-1}$. Esse achado corrobora os resultados de Schalch et al. (2005), que utilizaram banhos em solução aquosa na concentração de 2,0 mg. $\mathrm{L}^{-1}$, em 3 exposiçóes de 30 minutos de duração, a intervalos de 24 horas, com a mesma espécie de peixe e a mesma do parasito.
Uma explicação para o fraco desempenho do praziquantel e do levamisol é que ambos são solúveis em água. Dessa maneira, parte dos princípios ativos contidos na ração poderia ter se lixiviado (LAMMLER; GIESSEN, 1977). Por outro lado, no caso do diflubenzuron, embora a lixiviação possa ocorrer, sua eficácia é alta quando a temperatura da água varia de 20 a $25^{\circ} \mathrm{C}$ (PIRONET; JONES, 2000). Além disso, neste experimento, as drogas foram ministradas na ração e a ingestão do alimento foi rápida. Nesse caso a solubilidade ou náo em água parece desprovida de importância.

Outro ponto que pode ter prejudicado a eficácia dos tratamentos contra os 2 parasitos é o hábito alimentar onívoro da espécie de peixe ora utilizada. No momento da digestáo, ocorre ação de ácido clorídrico que permite a queda do $\mathrm{pH}$ da ingesta no estômago para melhor ação da pepsina (FURUYA, 1998). Dessa forma, a maioria dos medicamentos, na dependência de serem ácidos fracos ou bases, podem se dissociar trazendo variaçóes na quantidade de princípio ativo capaz de atravessar a fase lipídica das barreiras mucosas gastrintestinais (LAMMLER; GIESSEN, 1977).

Segundo Schaefer e Dupras (1976, 1977), o pó molhável (WP 25) não é persistente na água de viveiros devido à hidrólise e absorção pela matéria orgânica de fundo. $\mathrm{O}$ tratamento com diflubenzuron na forma de banhos para Ictalurus nebulosus e Pomoxis nigromaculatus não deixou resíduos nos tecidos após 7 dias (COLWELL; SCHAEFER, 1980). Schaefer e Dupras (1977) e Apperson et al. (1978) relataram que a quantidade de resíduos de diflubenzuron nos tecidos de Pomoxis annularis 
Tabela 2. Contagens de monogenóides e resultados das comparaçôes múltiplas e análise de variância. Jaboticabal, SP, Brasil.

\begin{tabular}{|c|c|c|c|c|c|c|c|c|}
\hline \multirow{2}{*}{ Tratamento } & \multicolumn{8}{|c|}{ Coletas / médias aritméticas ${ }^{1}$ de monogenóides / letras ${ }^{2}$} \\
\hline & \multicolumn{2}{|l|}{$\mathbf{1}^{\mathrm{a}}$} & \multicolumn{2}{|l|}{$2^{a}$} & \multicolumn{2}{|l|}{$3^{\mathrm{a}}$} & \multicolumn{2}{|l|}{$4^{a}$} \\
\hline 0 - controle & $85 \pm 60,9$ & $\mathrm{ab}$ & $178 \pm 109,1$ & $\mathrm{ab}$ & $166 \pm 83,0$ & $a b$ & $75 \pm 75,3$ & $\mathrm{ab}$ \\
\hline 2 - PZQ $952 m g$ + 1243 mg DFB & $62 \pm 32,2$ & $\mathrm{ab}$ & $72 \pm 8,8$ & $\mathrm{~b}$ & $40 \pm 9,2$ & $\mathrm{~b}$ & $24 \pm 8,1$ & $\mathrm{~b}$ \\
\hline 3 - PZQ 1952 mg + 935 mg DFB & $76 \pm 46,8$ & $\mathrm{ab}$ & $144 \pm 39,9$ & $\mathrm{ab}$ & $68 \pm 53,5$ & $\mathrm{ab}$ & $63 \pm 29,5$ & $\mathrm{ab}$ \\
\hline $5-$ LVM $380 \mathrm{mg}+949 \mathrm{mg}$ DFB & $102 \pm 61,3$ & $\mathrm{ab}$ & $112 \pm 81,2$ & $\mathrm{ab}$ & $73 \pm 88,3$ & $\mathrm{ab}$ & $68 \pm 41,7$ & $\mathrm{ab}$ \\
\hline $6-$ LVM $184 \mathrm{mg}+1237 \mathrm{mg}$ DFB & $189 \pm 50,8$ & $\mathrm{a}$ & $117 \pm 34,3$ & $a b$ & $68 \pm 13,7$ & $a b$ & $54 \pm 27,5$ & $\mathrm{ab}$ \\
\hline 7 - LVM $436 \mathrm{mg}+968 \mathrm{mg}$ DFB & $82 \pm 57,0$ & $\mathrm{ab}$ & $183 \pm 56,5$ & $\mathrm{ab}$ & $96 \pm 33,4$ & $\mathrm{ab}$ & $72 \pm 26,2$ & $\mathrm{ab}$ \\
\hline $8-$ LVM $436 \mathrm{mg}+1291 \mathrm{mg}$ DFB & $86 \pm 20,4$ & $\mathrm{ab}$ & $167 \pm 46,7$ & $\mathrm{ab}$ & $65 \pm 10,9$ & $\mathrm{ab}$ & $114 \pm 53,2$ & $\mathrm{a}$ \\
\hline 9 - DFB $935 \mathrm{mg}$ & $109 \pm 19,2$ & $\mathrm{ab}$ & $201 \pm 26,8$ & $\mathrm{a}$ & $128 \pm 26,2$ & $\mathrm{ab}$ & $109 \pm 60,2$ & $\mathrm{a}$ \\
\hline $13-\mathrm{LVM} 669 \mathrm{mg}+922 \mathrm{mg}$ PZQ & $41 \pm 20,9$ & $\mathrm{~b}$ & $103 \pm 3,2$ & $a b$ & $123 \pm 27,8$ & $\mathrm{ab}$ & $129 \pm 24,9$ & $\mathrm{a}$ \\
\hline 14 - LVM 438 mg + 1152 mg PZQ & $110 \pm 21,7$ & $\mathrm{ab}$ & $88 \pm 13,6$ & $a b$ & $141 \pm 65,3$ & $a b$ & $152 \pm 56,5$ & $\mathrm{a}$ \\
\hline 15 - PZQ $977 \mathrm{mg}$ & $60 \pm 32,0$ & $\mathrm{ab}$ & $99 \pm 55,4$ & $\mathrm{ab}$ & $139 \pm 50,9$ & $a b$ & $165 \pm 102,8$ & $\mathrm{a}$ \\
\hline 16 - PZQ $1964 \mathrm{mg}$ & $97 \pm 18,8$ & $\mathrm{ab}$ & $87 \pm 25,2$ & $\mathrm{ab}$ & $103 \pm 65,8$ & $\mathrm{ab}$ & $117 \pm 4,9$ & a \\
\hline 17 - LVM $362 \mathrm{mg}$ & $60 \pm 19,4$ & $a b$ & $107 \pm 22,7$ & $\mathrm{ab}$ & $196 \pm 54,5$ & $\mathrm{a}$ & $137 \pm 16,1$ & a \\
\hline 18 - LVM $640 \mathrm{mg}$ & $121 \pm 26,1$ & $\mathrm{ab}$ & $107 \pm 22,9$ & $\mathrm{ab}$ & $171 \pm 69,5$ & $\mathrm{ab}$ & $133 \pm 74,7$ & $\mathrm{a}$ \\
\hline \multicolumn{9}{|c|}{ Análise de variância ${ }^{2}$} \\
\hline Valor de F & 6,4 & & 6,62 & & 6,06 & & 7,02 & \\
\hline $\operatorname{Pr}>F^{*}$ & $<0,0001$ & & $<0,0001$ & & $<0,0001$ & & $<0,0001$ & \\
\hline
\end{tabular}

${ }^{1}$ Médias seguidas pela mesma letra, na coluna, não diferem entre si pelo teste de Scheffé $(P \geq 0,05)$.

${ }^{2} \mathrm{~A}$ análise de variância foi obtida após a transformação dos dados em $\log (\mathrm{x}+1)$.

*Probabilidade de significância do valor de F.

**Diferença mínima significativa, diflubenzuron - DFB, praziquantel - PZQ e levamisol - LVM.

Tabela 3. Número médio de Dolops carvalhoi antes e 3, 7 e 15 dias após tratamento de pacus (Piaratus mesopotamicus) com diferentes concentraçôes de diflubenzuron, praziquantel e levamisol adicionados na ração.

\begin{tabular}{|c|c|c|c|c|c|}
\hline & \multirow{2}{*}{$\begin{array}{c}\text { Tratamentos } \\
\text { (mg/kg de ração) }\end{array}$} & \multicolumn{4}{|c|}{$\begin{array}{l}\text { Número médio de parasitos } \\
\text { (D. carvalhoi) (em dias) }\end{array}$} \\
\hline & & antes & 3 & 7 & 15 \\
\hline $\mathrm{T}-1$ & PZQ $950+941 \mathrm{DFB}$ & 55 & 2 & 0 & 0 \\
\hline $\mathrm{T}-2$ & PZQ $952+1243$ DFB & 32 & 0 & 0 & 0 \\
\hline $\mathrm{T}-3$ & PZQ $1952+935$ DFB & 44 & 0 & 0 & 0 \\
\hline $\mathrm{T}-4$ & PZQ $2010+1273$ DFB & 56 & 0 & 0 & 0 \\
\hline $\mathrm{T}-5$ & LVM $380+949$ DFB & 53 & 2 & 0 & 0 \\
\hline T-6 & LVM 184 + 1237 DFB & 141 & 0 & 0 & 0 \\
\hline $\mathrm{T}-7$ & LVM $436+968$ DFB & 59 & 0 & 0 & 0 \\
\hline $\mathrm{T}-8$ & LVM 436 + 1291 DFB & 59 & 1 & 0 & 0 \\
\hline T-9 & DFB 935 & 58 & 0 & 0 & 0 \\
\hline $\mathrm{T}-10$ & DFB 1254 & 36 & 1 & 0 & 0 \\
\hline
\end{tabular}

$\mathrm{PZQ}=$ praziquantel, $\mathrm{LVM}=$ levamisol e DFB = diflubenzuron.
Tabela 4. Eficácia (\%) de diferentes concentraçóes de diflubenzuron, praziquantel e levamisol, simples e associado ao praziquantel e/ou levamisol no controle de Dolops carvalhoi avaliado 3, 7 e 15 dias após tratamento.

\begin{tabular}{llccc}
\hline \multirow{2}{*}{ Tratamentos } & \multicolumn{3}{c}{$\begin{array}{c}\text { Eficácia (\%) } \\
\text { dias de coleta }\end{array}$} \\
\cline { 3 - 5 } & & $\mathbf{3}$ & $\mathbf{7}$ & $\mathbf{1 5}$ \\
\hline T-1 & PZQ 950 + 941 DFB & 96,3 & 100 & 100 \\
T-2 & PZQ 952 + 1243 DFB & 100 & 100 & 100 \\
T-3 & PZQ 1952 + 935 DFB & 100 & 100 & 100 \\
T-4 & PZQ 2010 + 1273 DFB & 100 & 100 & 100 \\
T-5 & LVM 380 + 949 DFB & 96,2 & 100 & 100 \\
T-6 & LVM 184 + 1237 DFB & 100 & 100 & 100 \\
T-7 & LVM 436 + 968 DFB & 100 & 100 & 100 \\
T-8 & LVM 436 + 1291 DFB & 98,3 & 100 & 100 \\
T-9 & DFB 935 & 100 & 100 & 100 \\
T-10 & DFB 1254 & 97,2 & 100 & 100 \\
\hline
\end{tabular}

$\mathrm{PZQ}=$ praziquantel, $\mathrm{LVM}$ = levamisol e DFB = diflubenzuron. 
e Lepomis macrochirus foi 50 vezes superior à concentração na água, 4 dias após o tratamento, sendo rápida sua eliminação. $\mathrm{O}$ potencial de bioacumulação de diflubenzuron em $P$. annularis e L. macrochirus expostos à concentração de $10 \mathrm{ppb}$, durante 24 horas, foi de 822 e 848 ppb. A quantidade remanescente nos tecidos mostrou ser dependente da concentraçáo existente na água (SCHAEFER et al., 1979).

De acordo com Winkaler (2008), o diflubenzuron não é tóxico para o pacu, pois a aplicação de mais de $5000 \mathrm{mg} . \mathrm{L}^{-1}$ de água não causou qualquer tipo de sinal clínico. Porém reduziu a atividade da enzima glutationa transferase hepática, sugerindo interferência metabólica. A bioacumulação de diflubenzuron na musculatura de pacus foi 3,3 vezes maior que a concentração aplicada na água. Essa bioacumulação foi maior quando a droga foi aplicada na água do que quando ministrada pela ração. De acordo com Lopes (2005), essa bioacumulação no músculo de pacus foi 17 vezes superior à concentração na água nas primeiras 5 horas e, após esse período, não mais se observaram resíduos nas amostras de filés dos peixes.

Os resultados do presente trabalho permitem indicar, para o controle do D. carvalhoi, 3 a 7 dias de tratamento com diflubenzuron adicionado na ração, graças à sua eficácia e rápida eliminação nos tecidos do peixe. A droga pode ser utilizada na forma de péletes de ração para reprodutores, alevinos e peixes de engorda. Contudo, o tempo de carência do produto deve ser respeitado para garantir segurança alimentar. Seu uso deve ser parcimonioso, levando-se em conta a proximidade do abate para consumo e o uso de quarentenários isolados do ambiente de criação, considerando-se seus efeitos sobre invertebrados planctônicos que poderiam prejudicar a cadeia alimentar.

\section{Conclusóes}

A utilização do diflubenzuron associado ou não ao levamisol e ao praziquantel na ração, foi eficaz no controle de D. carvalhoi nas condiçôes deste ensaio. Não foi observada eficácia terapêutica adequada, quando se utilizou o levamisol, praziquantel e diflubenzuron simples ou associados no controle de $A$. penilabiatus.

\section{Referências}

APPERSON, C. S. et al. Effects of diflubenzuron on Chaoborus astictopus and nontarget organisms and persistence of diflubenzuron in lentic habitats. Journal of Economic Entomology, v. 71, n. 1, p. 521-527, 1978.

COLWELL, A. E.; SCHAEFER, C. H. Diets of Ictalurus nebulosus and Pomoxis nigromaculatus altered by diflubenzuron. Canadian Journal of Fisheries and Aquatic Sciences, v. 37, n. 5, p. 632-639, 1980.

EIRAS, J. C. Elementos de ictioparasitologia. 1 ed. Porto: Fundação Eng. Antônio de Almeida, 1994. 339 p.

EISLER, R. Diflubenzuron hazards to fish, wildlife and invertebrates: a synoptic review. U.S. Fish Wildlife Service and Biological Report, v. 4, n. 25, p. 1-36, 1992.

ERDAL, J. I. New drug treatment hits sealice when they are most vulnerable. Fish farming International, v. 24, n. 2, p. 1-9, 1997.
FURUYA, W. M. Nutriçáo de peixes: Atualização em piscicultura de água doce. Módulo - III. Maringá: Editora e Gráfica Cotação, 1998. p. 1-22.

GUSTAFSSON, L. L.; BEERMAN, B.; ABDY, Y. A. Handbook of drugs for tropical parasitic infections. London: Ed. Taylor \& Francis, 1987. $151 \mathrm{p}$.

HOSBERG, T. E.; HOY, T. Tissue distribution of carbon-14 diflubenzuron in Atlantic salmon (Salmo salar). Acta Veterinaria Scandinavica, v. 32, n. 4, p. 527-533, 1991.

HIRAZAWA, N.; OHTAKA, T.; HATA, K. Challenge trials on the anthelmintic effect of drugs and natural agents against the monogenean Heterobothrium okamotoi in the tiger puffer Takifugu rubripes, Oita, Japan. Aquaculture, v. 188, n. 1-2, p. 1-13, 2000.

LAMMLER, G.; GIESSEN, D. Agentes Antiparasitários. In: FRIMMER, M.; LÄMMLER, G. Farmacologia e Toxicologia em Veterinária. Rio de Janeiro: Guanabara Koogan, 1977. Cap.5, p. 59-68.

LITTLE, T. M.; HILLS, F. J. Agricultural experimentations desings and analysis. New York: Wiley, 1978. 350 p.

LOPES, R. B. Análise ecotoxicológica dos xenobióticos triclorfon e diflubenzuron empregados na aquicultura continental. Piracicaba, 2005. 104 f. Tese (Doutorado em Ciências-Energia Nuclear na Agricultura) - Universidade de São Paulo.

MARTINS, M. L.; ROMERO, N. G. Efectus del parasitismo sobre el tegido branquial en peces cultivados : Estudio parasitológico e histopatológico. Revista Brasileira de Zoologia, v. 13, n. 2, p. 489-500, 1996.

MORAES, F. R.; MARTINS, M. L. Condiçóes pré-disponentes e principais enfermidades de teleósteos em piscicultura intensiva. In: CYRINO, J. E. P. et al. (ed). Tópicos especiais em piscicultura de água doce tropical intensiva. São Paulo: TecArt, 2004. Cap.3, p. 343-386.

NOGA, E. J. Fish Disease. Diagnosis and Treatment. St. Louis: MosbyYear Book, 1996. 367 p.

PAVANELlI, G. C.; EIRAS, J. C.; TAKAMOTO, R. M. Doenças de peixes: Profilaxia, Diagnóstico e Tratamento. Maringá: Eduem, 2002. 305 p.

PELLITERO, P. A. Enfermidades producidas por parasitos en peces, In: MONTEROS, J. E.; LABARTA, U. Patología en acuicultura. Madrid: Mundi-Prensa Libros, 1988. p. 215-316.

PIRONET, F. N.; JONES, J. B. Treatments for ectoparasites and diseases in captive Western Australian dhufish. Aquaculure International, v. 8, n. 4, p. 349-361, 2000.

REDMAN, C. A. et al. Praziquantel: an urgent and exciting challenge. Parasitology Today, v. 12, n. 1, p. 14-20, 1996.

ROBERTSON, S. J.; MARTIN, R. J. Levamisole-activated singlechannel currents from muscle of the nematode parasites Ascaris suum. British Journal of Pharmacology, v. 108, n. 1, p. 170-178, 1993.

Rhode, K. Ecology of marine parasites. Wallingford: CAB International, 1993. 298 p.

SAS INSTITUTE INCORPORATION. The SAS-System for Windows release 8.2 (software). Cary, 1999-2001.

SCHALCH, H. C. S. et al. Eficácia do diflubenzuron no controle de Dolops carvalhoi (Crustacea: Branchiura) em jovens pacus Piaractus mesopotamicus Holmberg 1887 (osteichthyes: characidae) naturalmente infestados. Acta Scientiarum Animal Sciences, v. 27, n. 2, p. 297-302, 2005. 
SCHAEFER, C. H.; DUPRAS Jr, E. F. Factors affecting the stability of Dimilin in water and the persistence of Dimilin in field waters. Journal of Agricultural and Food Chemistry, v. 24, n. 4, p. 733 - 739, 1976.

SCHAEFER, C. H.; DUPRAS Jr, E. F. Residues of diflubenuron [1-(4-Chlorophenyl)-3-(2,6-difluorobenzoyl)urea] in pasture soil, vegetation, and water falling aerial application. Journal of Agricultural and Food Chemistry, v. 25, n. 5, p. 1026-1030, 1977.

SCHAEFER, C. H. et al. The accumulation and elimination of diflubenzuron by fish. Bulletin of Environmental Contamination and Toxicology, v. 21, n. 1, p. 249-254, 1979.

SCHMAHL, G.; MEHLHORN, H. Treatment of fish parasites. Zeitschrift für Parasitenkunde, v. 71, n. 6, p. 727-737, 1985.

SHIMURA, S. Seasonal occurrence, sex ratio and site preference of Argulus coregoni Thorell (Crustacea:Branchiura parasitic on cultured freshwater salmonids in Japan. Parasitology, v. 86, n. 3, p. 537-552, 1983.
SIPAÚBA-TAVARES, L. H. Limnologia Aplicada à Aquicultura. Jaboticabal: FUNEP, 1995. 70 p.

STEPHENS, F. J. et al. Treatments to control Haliotrema abaddon in the West Australian dhufish, Glaucosoma hebraicum. Aquaculture, v. 215, n. 1, p. 1 - 10, 2003.

TANNER, D. K.; MOFFETT, M. F. Effects of diflubenzuron on the reproductive success of the bluegill sunfish, Lepomis macrochirus. Environmental Toxicology and Chemistry, v. 14, n. 8, p. 1345-1355, 1995.

THATCHER, V. E. Amazon Fish Parasites. Amazoniana, v. 11, n. 3-4, p. 280-281, 1991.

WINKALER, E. U. Análises de resíduos, riscos alimentares, e variáveis bioquímicas de pacu Piaractus mesopotamicus exposto ao diflubenzuron e teflubenzuron. Jaboticabal, 2008. $60 \mathrm{f}$. Tese (Doutorado em Aquicultura) - Centro de Aquicultura, Universidade Estadual Paulista. 